\title{
Application of Wire-Assisted Reduction Guided by Minimally Invasive Wire Introducer in the Treatment of Irreducible Femoral Intertrochanteric Fractures
}

\section{Xiaocong Lin}

the second affiliated hospital of fujian medical university

\section{Xiuxi Huang}

the second affiliated hospital of fujian medical university

Kaibin Fang

the second affiliated hospital of fujian medical university

Qingfeng Ke

the second affiliated hospital of fujian medical unibersity

\section{Shaojian Shi}

the second affiliated hospital of fujian medical university

Zhangsheng Dai ( $\nabla$ 1009828183@qq.com )

the second affiliated hospital of fujian medical university

\section{Research article}

Keywords: irreducible femoral intertrochanteric fractures, wire-assisted reduction, wire introducer

Posted Date: August 21st, 2020

DOl: https://doi.org/10.21203/rs.3.rs-51793/v1

License: (1) This work is licensed under a Creative Commons Attribution 4.0 International License.

Read Full License 


\section{Abstract}

Background: Open reduction was often required in the treatment of irreducible femoral intertrochanteric fractures. A minimally invasive wire introducer was designed to assist the reduction of such fractures. The aim of our study was to investigate the clinical outcomes of this technique.

Methods: Between 2013 and 2018, 92 femoral intertrochanteric fracture patients who were treated with intramedullary nail fixation and difficult reduction using the traction beds were retrospectively reviewed. Decision for surgery was based on the displacement of the fracture. The patients was divided into two groups, 31 in the control group and 61 in the observation group. The control group were received open reduction operation. And the observation group still received the closed reduction operation, using minimally invasive wire introducer to guide the wire and assist the fracture reduction. The operation time, blood loss, VAS scores, angulation, reduction, neck-shaft angle, redisplacement, limb length discrepancy, and union time were recorded.

Results: All patients of two groups were successfully operated and were followed up for an average of 23.8 months. There was no statistical difference in baseline data between the two groups. The observation group had shorter operation time, lower VAS score and less intraoperative bleeding. And the datas are statistically different.

Conclusion: Minimally invasive wire introducer is a good technology to guide the wire for irreduciblr femoral intertrochanteric fracture reduction. Using this technique, irreduciblr femoral intertrochanteric fractures could be restored and good clinical outcome was achieved.

Level of evidence: Level IV; Case Series; Treatment Study

\section{Introduction}

With the aging of the social population, femoral intertrochanteric fractures have become one of most common fractures. If these patients received conservative treatment, they may need to stay in bed for a long time. Prolonged bedridden could lead to pressure sores, pneumonia, deep vein thrombosis and other complications, and can lead to death. So the intertrochanteric fracture was called the "last fracture of life" [1]. Therefore, surgical treatment was the preferred choice for intertrochanteric fractures. Intramedullary nail fixation has been widely used in recent years due to its advantages of minimally invasive [2].

However, 3-17\% of intertrochanteric fractures were still difficult to be closure reduced by traction bed [3]. These fractures were commonly referred to irreducible intertrochanteric fractures. Techniques such as warp by kirschner wire prick, bone hook, and clamp were often used in the reduction of these fractures[46]. The above methods had several disadvantages. The first was the incision need to be enlarged. The second was that these methods sometimes required the surgeons to manually maintained the reduction of the fractures, and surgeons and patients were exposed to more radiation. Stable clinical immobilization was also often difficult to obtain using the above methods. In order to reduced the trauma 
of the patients and maintained stable reduction of the fractures, minimally invasive wire introducer was designed and used for intramedullary nail fixation surgery. The clinical outcomes of this technology was applied in the current study.

\section{Materials And Methods}

\section{Study design and patients}

From 2013 to 2018, femoral intertrochanteric fracture patients who were treated with intramedullary nail fixation in our hospital were included in this study. All these patients were hospitalized for trauma. The causes of trauma were traffic accidents or falls. Average time from injury to operation was 2 days (range 1-5 days). Patients were initially included in the study when their fractures were difficult reduction using the traction beds. The inclusion criteria were older than 60 years and the patient had no prior history of hip surgery. The study excluded patients with open fracture or pathological fractures. Patients with vascular or nerve damage were also excluded from the study.There were no obvious medical complications or inflammatory joint disease in these cases.

The general data of the two groups of patients were collected before the operation. 92 patients were included in the study, including 31 cases in the observation group and 61 cases in the control group.

\section{Surgical techniques}

Surgery was performed on a traction bed under spinal anaesthesia. Longitudinal traction was applied to the fractured limb with the traction bed. After the closed reductions were failured, the patients were randomly divided into two groups. The control group received open reduction, with a small incision designed at the fracture region. Then tools such as bone hooks and steel wire were used to assist the reduction. The observation group still received the closed reduction operation. The minimally invasive wire introducer were used to reduce fractures in the observation group. The front and lateral skin projection points of the intertrochanteric fracture was located by $\mathrm{C}$-arm $\mathrm{X}$ ray. At these two points made two small incisions about $0.6 \mathrm{~mm}$. Using the wire introducer (semicircular diameter $120 \mathrm{~mm}$ ) punctured in from the front incision, through the medial and the posterior femur fracture, then poked out from the lateral incision under fluoroscopic control. The wire go around the femur fracture using this introducer. The second wire introducer punctured in from the lateral incision, through the front of the femur fracture, then poked out from the front incision. Two introducer should go through the same channel in muscle as much as possible and should go through the femur surface as close as possible. The wire got in the tip of the second wire introducer, then got out from the lateral incision guide by the inroduce.The intertrochanteric fracture was reduced when the wire was tighten from lateral incision under fluoroscopic control. The intramedullary nail was inserted using percutaneous technique. The head screw was placed in keeping with the tip apex distance (TAD) should be $<25 \mathrm{~mm}$ to minimise the risk of cutout and in the middle-middle or middle-inferior position. The standard implant for intramedullary nail was a nail of 170$200 \mathrm{~mm}$ in length and $9-11 \mathrm{~mm}$ in diameter. 
Figure1a: Design drawing of wire guide device; b:Three dimensional design drawing of wire guide device; c:A 49 years old male patient underwent surgical treatment for the left femural intertrochanteric fracture; $\mathrm{d}$ :Place the wire with the assist of the wire guide; e:Intraoperative fluoroscopy indicated good position; $\mathrm{f}$ :Postoperative X-ray showed that the reduction of fracture was good; g:Three months after operation, reexamination indicated fracture healing(see attached)

\section{Postoperative care}

The first day after surgery, the patients underwent a routine blood test to estimate the blood loss. After surgery, patients could begin quadriceps contraction exercises and ankle pump training under the guidance of a physician. If the patient's condition permits, the patient can sit up. On the second day after surgery, the patient received wound cleaning and X-ray examination. They received continuous followed at 4 weeks, 8 weeks, 12 weeks, 6 months and 12 months. Patients were followed up for X-ray examination to see if the fracture were healed. If there is no sign of fracture healing at 12 week, the patient will be followed up every 3 weeks. Patients could walk with the assist of crutches with no load.After X-ray identification of fracture healing, the patients would get the permission of full activities and weightbearing.

\section{Contrast index}

Visual analogue scale (VAS) was used to evaluate postoperative pain. Two orthopedic physicians evaluated the postoperative imaging data of patients, and they did not know the evaluation results estimated by each other. If the results of the two evaluations are different, a chief orthopaedic physician will conduct the final evaluation. Maximum cortical displacement and angulation at fracture site on antero-posterior (AP) and lateral radiographs were used to evaluate the reduction. According to the above indexes, we divided the reduction of fracture into four grades. When the angle of the fracture was less than $10^{\circ}$ and the maximum displacement distance of the fracture was less than $4 \mathrm{~mm}$, the fracture was defined as good reduction. When the the fracture was more than $10^{\circ}$ or the maximum displacement distance of the fracture was more than $4 \mathrm{~mm}$, the fracture was defined as acceptable reduction. When the the fracture was more than $10^{\circ}$ and the maximum displacement distance of the fracture was more than $4 \mathrm{~mm}$, the fracture was defined as poor reduction[7]. Limb length discrepancy(LLD) was measured by comparing the length of the lower extremities of opposite side. When LLD was more than $1 \mathrm{~cm}$, it could be considered that LLD was significant[8]. When there was no tenderness in the fracture area and callus formation could be seen on the X-ray, the fracture was considered to be healed. Neck-shaft angle was gauged by the postoperative radiograph and was compared with the opposite side. When the angle difference between the uninjured hip joint and the operative hip joint was more than $5^{\circ}$, reduction was considered as varus reduction. The patient's activity and complications were recorded during follow-up. This comparison project has been approved by the ethics committee of our hospital.

\section{Statistical analysis}

Statistical assessment was performed using SPSS V19 software. Consecutive data were summarized as mean and standard deviation (SD), or median and range; whereas categorical data were summarized as 
frequencies and percentage. Comparison amongcategorical variables was performed using Chi-Square test; for continuous data, independent t-test was used if variables were normally distributed, otherwise Mann-Whitney test was employed.

\section{Results}

All patients were followed up for an average of 23.8 months. As mentioned above, 92 patients were included in the study, including 31 cases in the observation group and 61 cases in the control group. In general, there were no statistically significant differences to the baseline demographic factors between the two groups(See in Table 1).

All patients of two groups were successfully operated.No patient died during the perioperative period. The observation group had shorter operation time, lower VAS score and less intraoperative bleeding. Patients in the observation group also had shorter fracture healing time. And the datas were statistically different. There was no statistical difference in the angulation, reduction, neck-shaft angle and LLD between two groups. One patient in the observation group received revision surgery while three in the control group. One patient in the observation group had poor reduction, while three in the control group. There was no statistical difference in these datas.(See in Table 2).

Table 1

Baseline demographic characteristics of study patients

\begin{tabular}{|llll|}
\hline Variables & Observation group & control group & P value \\
\hline Age(years) & $63.53 \pm 11.27$ & $66.72 \pm 13.56$ & 0.26 \\
\hline Sex(Male/Female) & $12 / 19$ & $33 / 28$ & 0.16 \\
\hline Fracture classification(AO classification) & & & 0.65 \\
A1 & 9 & 15 & 0.53 \\
A2 & 15 & 29 & 0.23 \\
A3 & 7 & 17 & 0.26 \\
\hline Neck-shaft angle of uninjured hip $\left(^{\circ}\right)$ & $132.73 \pm 3.33$ & $133.16 \pm 2.92$ & \\
Time from injury to surgery (days) & $1.15 \pm 0.72$ & $1.33 \pm 0.66$ & \\
Maximum cortical displacementa(mm) & $1.15 \pm 0.36$ & $1.03 \pm 0.53$ & \\
\hline
\end{tabular}


Table 2

Summarized depiction of perioperative data and results

\begin{tabular}{|llll|}
\hline Parameters & Observation group & control group & P value \\
\hline The operation time(minute) & $73.53 \pm 13.63$ & $101.26 \pm 22.79$ & 0.00 \\
\hline Blood loss $(\mathrm{ml})$ & $135.26 \pm 25.33$ & $223.76 \pm 37.55$ & 0.00 \\
VAS scores on the first day after surgery & $3.72 \pm 0.91$ & $5.15 \pm 1.26$ & 0.00 \\
\hline Angulation $\left(^{\circ}\right)$ & $3.16 \pm 0.37$ & $3.23 \pm 0.62$ & 0.57 \\
Reduction $(\%)$ & 26 & 50 & 0.82 \\
Good & 2 & 5 & 0.62 \\
Acceptable & 3 & 6 & 0.62 \\
poor & $133.92 \pm 2.96$ & $134.29 \pm 3.53$ & 0.36 \\
Neck-shaft angle (\%) & $1.19 \pm 0.37$ & $1.13 \pm 0.61$ & 0.38 \\
Neck-shaft angle of operated hip & 1 & 5 & 0.71 \\
Difference with uninjured hip & 3 & 3 & 0.00 \\
Cases of poor reduction & 1 & 3 & 0.71 \\
Varus reduction $\left(>5^{\circ}\right)$ & $13.16 \pm 3.71$ & $15.96 \pm 3.65$ & \\
Redisplacement at follow-up $(>5 \mathrm{~mm}$ ) & 1 & 3 & \\
LLD (> 1 cm, & & & \\
Union time (week) & & & \\
Reoperation & & & \\
\hline
\end{tabular}

\section{Discussion}

Reduction was the key factor to determine the operative effect of intertrochanteric fracture of femur[9]. Many complications, such as hip varus, loosening of internal fixation, and refracture, were associated with poor reduction[10]. Poor reduction was more common in irreducible fractures. Helin reported that when PFNA was used to treat unstable intertrochanteric fractures in the elderly, the incidence of fracture dislocation was as high as $45 \%$, and the femoral neck was shortened by nearly $9 \mathrm{~mm}$ after reduction[11]. Cho also confirmed that the load at the intertrochanteric fracture was related to the reduction of the fracture through the study of biomechanics. Whether the reduction was good or not directly determines whether the patient could get out of bed early to reduce bed-related complications[12].

Traction bed was widely used in the reduction of intertrochanteric fractures[13]. The closed reduction under traction was to tighten the muscle and soft tissue of the proximal end of the femur after the longitudinal traction of the distal end of the fracture, and to achieve the reduction of the fracture end 
through the splint action of the muscle, ligament and joint capsule. There were many muscles attached to the proximal femur, which had different directions of action on the bone. Different fractures were accompanied by different injury mechanisms, and the muscle forces were also different. Simple longitudinal traction did not always succeed in fracture reduction. In order to achieve good reduction of fracture, some patients still needed to receive open reduction. For these patients, it was very important to minimize the incision and the extent of soft tissue peeling on the basis of good reduction. It was a common clinical method to maintain reduction and fixation of fracture with steel wire, which had the advantages of simple application and firm fixation[14]. After open reduction, the fracture could be maintained by using the wire, which was stable but had significant soft tissue damage and affected bone healing[15].

To minimize soft tissue injuries, we designed this minimally invasive wire guide. This device had several advantages. First, the operation only needed two extra small incisions(about 5 millimeters) on the skin and deep fascia, so there was no serious soft tissue injuries. With the assist of this device, the fracture could be encircled by a wire, and the fracture reduction was stable to prepare for the next step of fixation. Apivatthakakul T reported that the use of minimally invasive methods did not affect the blood supply of femoral[16]. Our research also confirmed this. In our study, patients who received wire placement with minimally invasive guides had shorter healing times. These patients also had less intraoperative blood loss. They also reported less pain, which suggests that the device is less invasive. This device was easy to use. The shorter operative time in the observation group supported the above statement.

There were still some notices when using this device. There were many important vessels and nerves around the femur that must not be damaged. Because the distal end of this instrument was sharp, the distal end must be kept close to the bone when the instrument was moved around the femur. Generally, we draw a line from the anterior superior iliac spine to the lateral margin of the patella. The incision where the wire guide placed was built on this line and were located by $\mathrm{C}$-arm $\mathrm{X}$ ray. These procedures could avoid vessels and nerves injuries. The muscles needed to be protected, when using the device, especially the gluteus medius[17]. In most cases, our study suggests that this technique may allow patients to achieve the same reduction as an open reduction. The incidence of complications and malreduction did not increase.

More cases and longer follow-up are still needed to confirm our results. Existing study suggests that the wire guide designed by us can achieve an effect similar to that of open reduction for difficult reduction of intertrochanteric fractures with less trauma. Minimally invasive wire introducer is a good technology to guide the wire for reset. Using this technique, irreduciblr femoral intertrochanteric fractures can be restored and good clinical outcome is achieved.

\section{Declarations}

\section{Declarations}


This study was approved by the institutional review board at The Second Affiliated Hospital of Fujian Medical University (\#2020.183). This study has been performed in accordance with the Declaration of Helsinki.Written informed consent was obtained from all the participants in this study.

\section{Consent to publish}

Not applicable.

\section{Availability of data and materials}

The datasets used and/or analyzed during current study are available from the corresponding author on reasonable request.

\section{Competing interests}

The authors declare that they have no conflict of interest

\section{Funding}

This study did not receive any specific grant from funding agencies in the public, commercial, or not-forprofit sectors.

\section{Author's contributions}

Study design: FKB. Study conduct:LXC.Data interpretation: SSJ. Drafting manuscript: HXX,KQF.DZS takes responsibility for the integrity of the data analysis.

\section{Acknowledgements [}

We appreciated Yizhong Li for technological guidance of the manuscript.

\section{References}

1】 Sellan M, Bryant D, Tieszer C,.et al. Short Versus Long InterTAN Fixation for Geriatric Intertrochanteric Hip Fractures: A Multicentre Head-to-Head Comparison[J]. Journal of orthopaedic trauma, 2019, 33(4):169.

2】 Constantine Kokoroghiannis, loannis Aktselis, Anastasios Deligeorgis,et al. Evolving concepts of stability and intramedullary fixation of intertrochanteric fractures-A review[J]. Injury, 43(6):686-693.

3】 Michael H. Amini, John J. Feldman, John C. Weinlein. High Complication Rate in Young Patients With High-Energy Intertrochanteric Femoral Fractures[J]. Orthopedics, 2016, 40(2):1.

4】 Parsch, Klaus, Weller, Svenja, Parsch, Dominik. Open Reduction and Smooth Kirschner Wire Fixation for Unstable Slipped Capital Femoral Epiphysis[J]. Journal of Pediatric Orthopedics, 29(1):1-8. 
5区 Olsson O, Ceder L, Hauggaard A. Femoral shortening in intertrochanteric fractures. A comparison between the Medoff sliding plate and the compression hip screw.[J]. 2001, 83(4):572.

6】 K. M. Fox, S. R. Cummings, E. Williams,et al.. Femoral Neck and Intertrochanteric Fractures Have Different Risk Factors: A Prospective Study[J]. Osteoporosis International, 11(12):1018-1023.

7囚 Hoskins W, Bingham R, Joseph S, et al. Subtrochanteric fracture: the effect of cerclage wire on fracture reduction and outcome. Injury. 2015;46:1992e1995

8区 Todd M. Chappell, Casey C. Ebert, Kevin M. McCann,et al. Distal tibial distraction osteogenesis-an alternative approach to addressing limb length discrepancy with concurrent hindfoot and ankle reconstruction[J]. Journal of Orthopaedic Surgery and Research, 2019, 14(1).

98 Parry JA, Barrett I, Schoch B,et al. Does the Angle of the Nail Matter for Pertrochanteric Fracture Reduction? Matching Nail Angle and Native Neck-Shaft Angle[J]. Journal of Orthopaedic Trauma, 2018, 32(4):174.

10区 Sellan M, Bryant D, Tieszer C, et a. Short Versus Long InterTAN Fixation for Geriatric Intertrochanteric Hip Fractures: A Multicentre Head-to-Head Comparison[J]. Journal of orthopaedic trauma, 2019, 33(4):169.

11】 Hélin M, Pelissier A, Boyer P, et al. Does the PFNATM nail limit impaction in unstable intertrochanteric femoral fracture? A 115 case-control series. Orthop Traumatol Surg Res, 2015, 101(1): 4549.

12】 Cho SH, Lee SH, Cho HL, et al. Additional fixations for sliding hip screws in treating unstable pertrochanteric femoral fractures (AO Type 31-A2): short-term clinical results. Clin Orthop Surg, 2011, 3(2): 107-113.

13囚 Yoon, Pil Whan, Kwon, Ji Eun, Yoo, Jeong Joon,et al. Femoral Neck Fracture After Removal of the Compression Hip Screw From Healed Intertrochanteric Fractures[J]. Journal of Orthopaedic Trauma, 27(12):696-701.

14】 Akhil A Tawari, Harish Kempegowda, Michael Suk, et al. What Makes an Intertrochanteric Fracture Unstable in 2015? Does the Lateral Wall Play a Role in the Decision Matrix?[J]. Journal of Orthopaedic Trauma, 2015, 29 Suppl 4:S4-S9.

15》 Kleweno, Conor, Morgan, Jordan, Redshaw, James, et al. Short Versus Long Cephalomedullary Nails for the Treatment of Intertrochanteric Hip Fractures in Patients Older than 65 Years[J]. Journal of Orthopaedic Trauma, 28(7):391-397.

16ه Akhil A Tawari, Harish Kempegowda, Michael Suk,et al. What Makes an Intertrochanteric Fracture Unstable in 2015? Does the Lateral Wall Play a Role in the Decision Matrix?[J]. Journal of Orthopaedic Trauma, 2015, 29 Suppl 4:S4-S9. 
17囚 John E Arvesen, Stephen W Gill, Philip M Sinatra,et al. Biomechanical Contribution of TensionReducing Rotator Cuff Sutures in 3-Part Proximal Humerus Fractures[J]. Journal of Orthopaedic Trauma, 2016, 30(8).

\section{Figures}
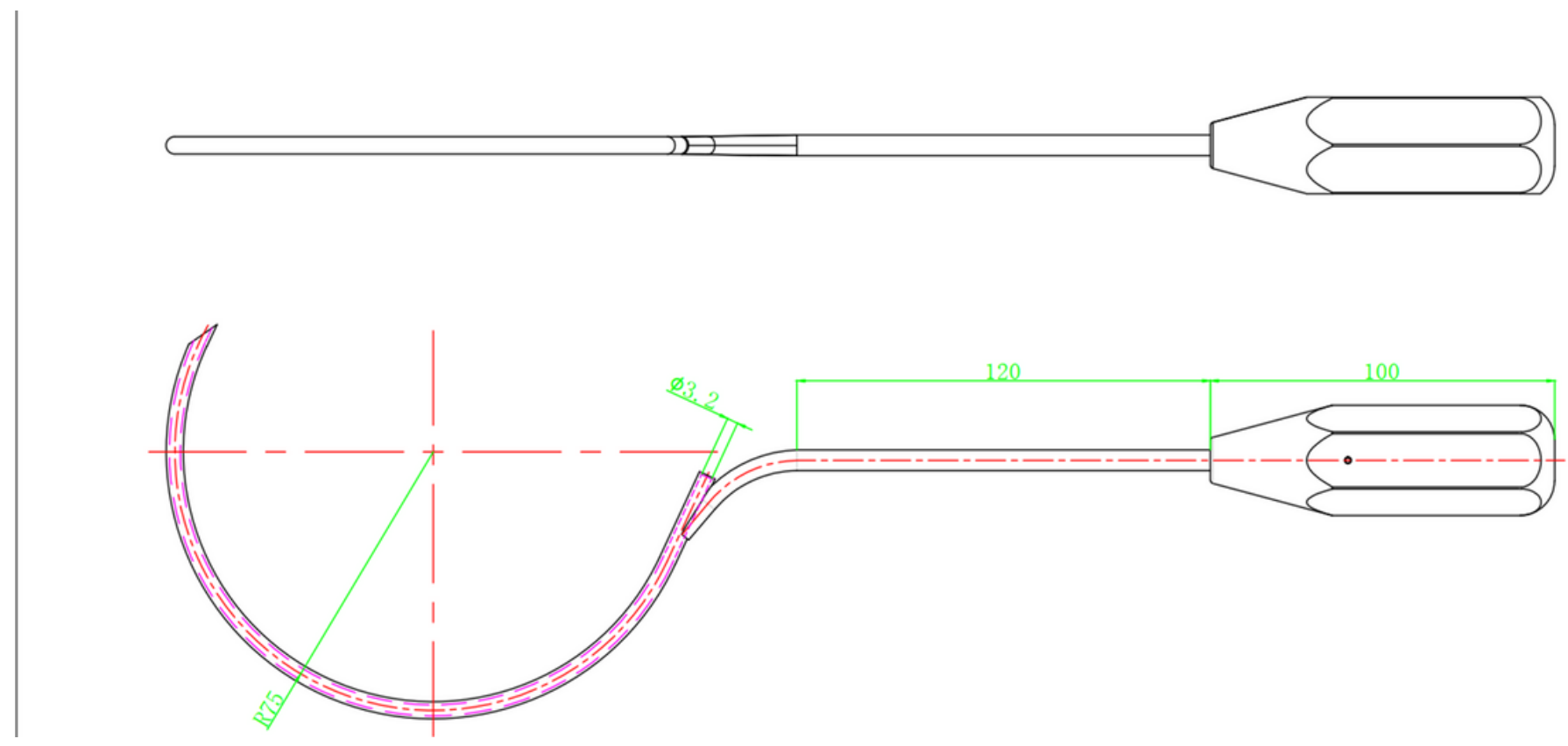

\section{Figure 1}

Design drawing of wire guide device;

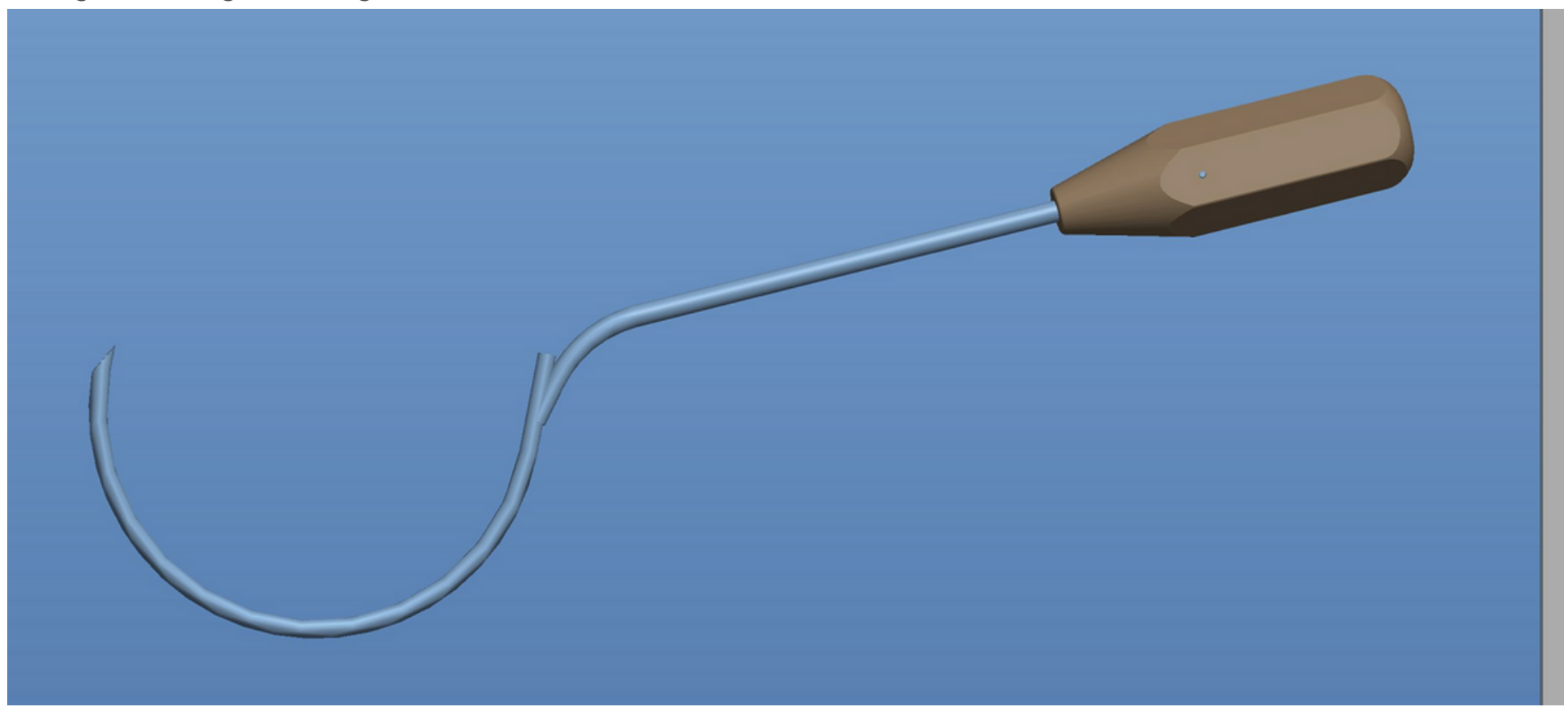


Figure 2

Three dimensional design drawing of wire guide device;

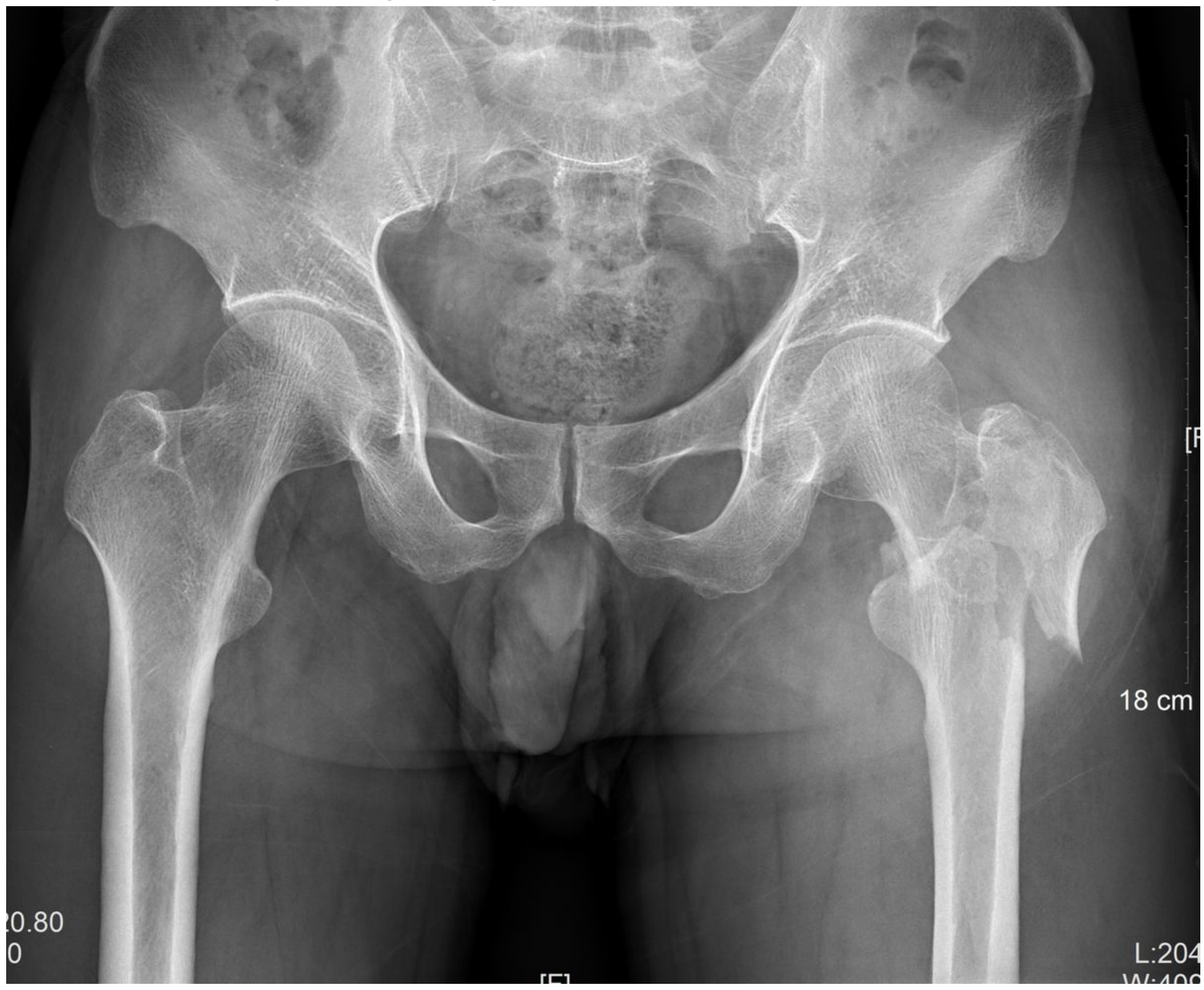

Figure 3

A 49 years old male patient underwent surgical treatment for the left femural intertrochanteric fracture 


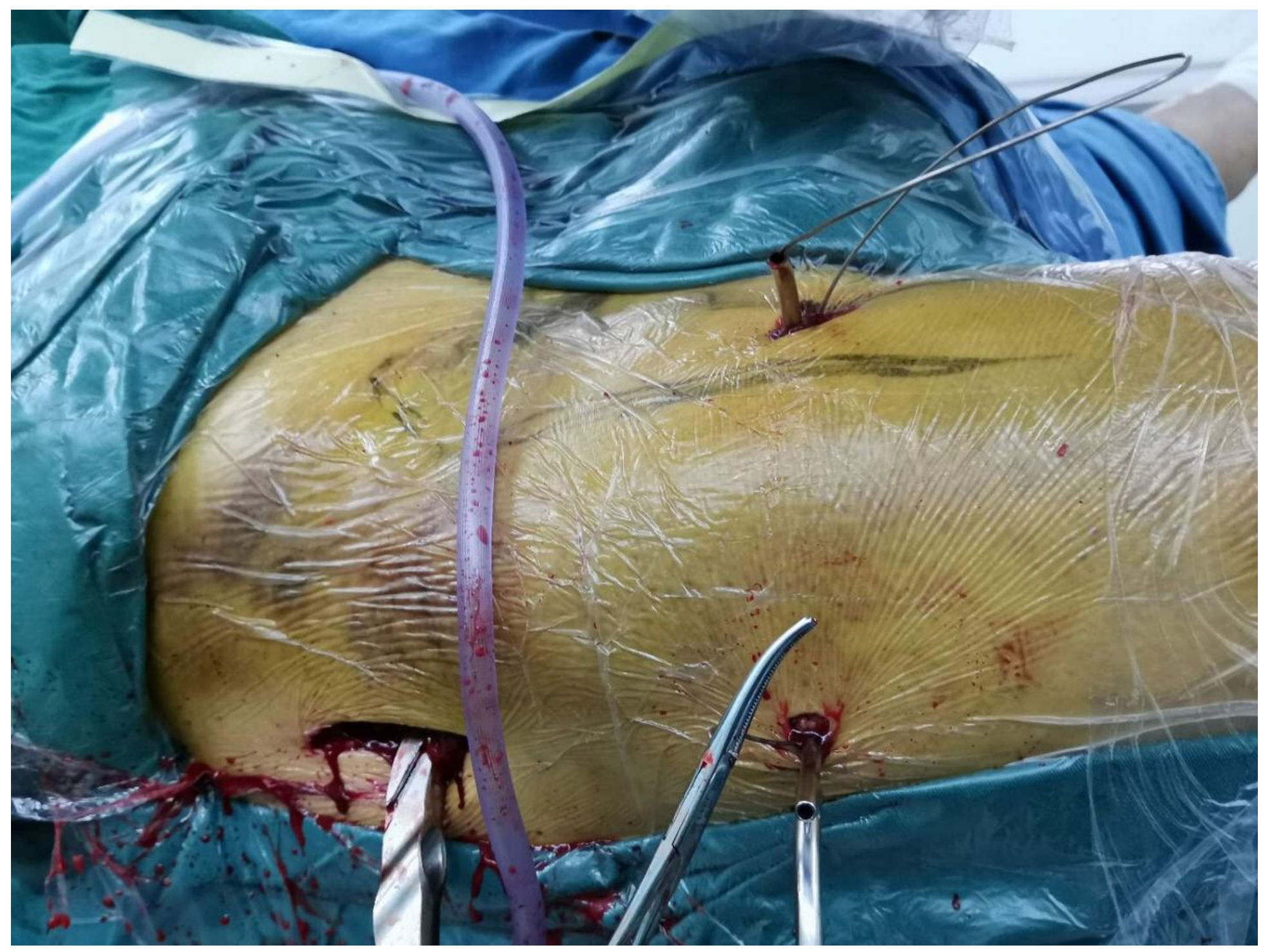

Figure 4

Place the wire with the assist of the wire guide 


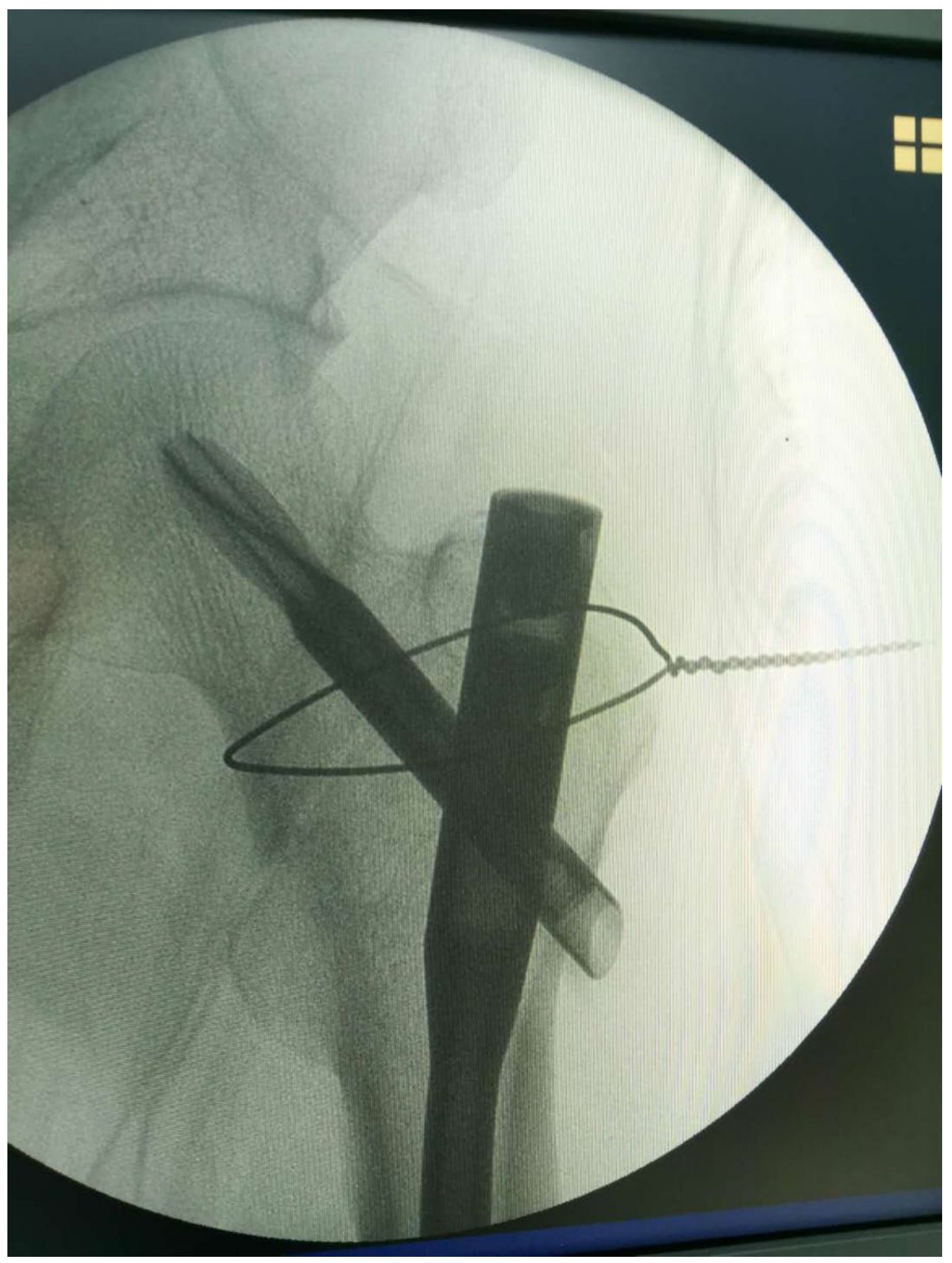

Figure 5

Intraoperative fluoroscopy indicated good position 


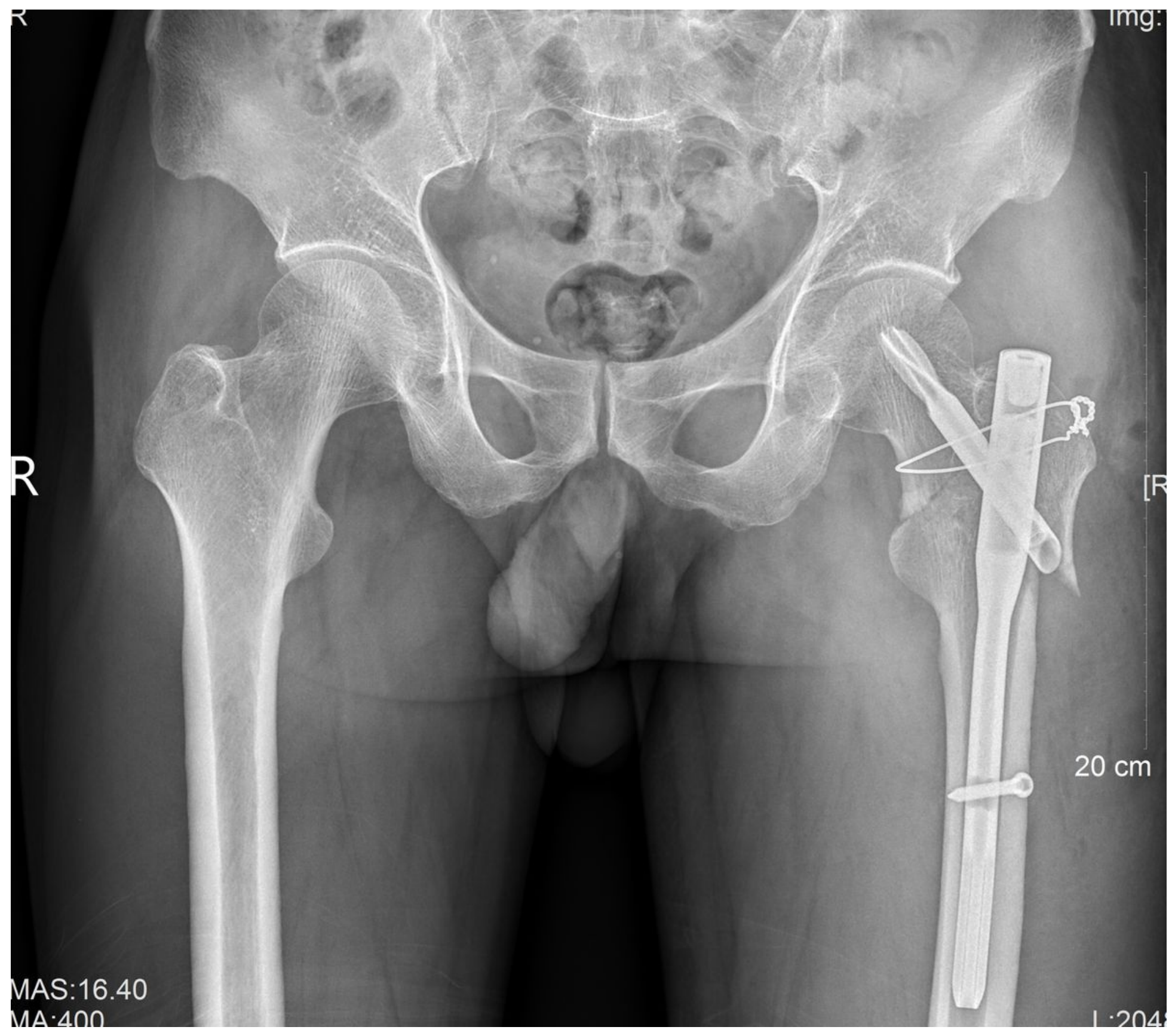

Figure 6

Postoperative X-ray showed that the reduction of fracture was good 


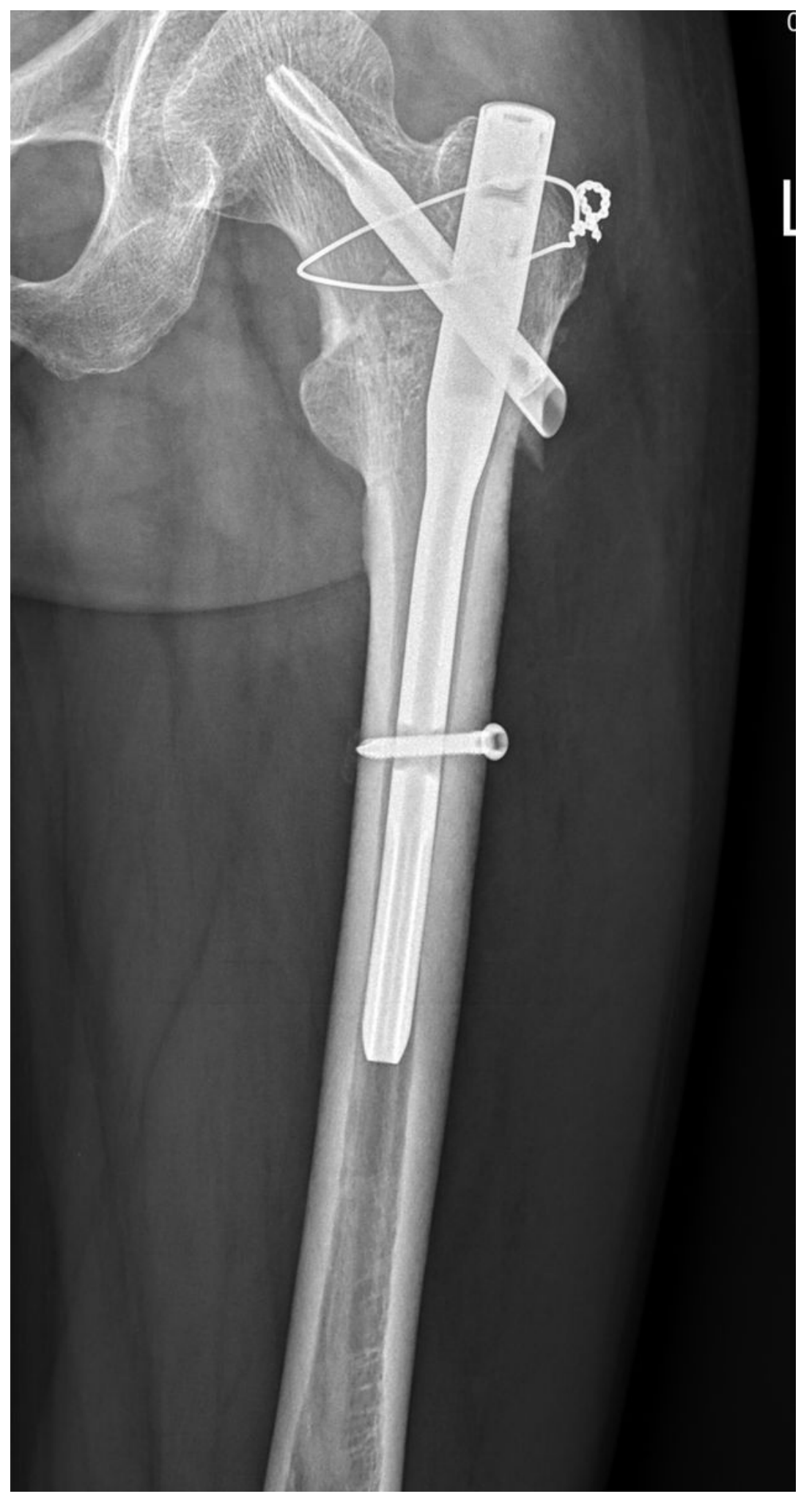

\section{Figure 7}

Three months after operation, reexamination indicated fracture healing(see attached) 